\title{
DYNAMIC ECONOMIC DISPATCH USING MODIFIED BACTERIAL FORAGING ALGORITHM ORIENTED WITH WEIGHTED PARTICLE SWARM OPTIMIZATION
}

\author{
Ehab E. Elattar \\ Department of Electrical Engineering, Faculty of Engineering, \\ Menoufia University, Shebin El-Kom, Egypt
}

\begin{abstract}
The goal of dynamic economic dispatch (DED) is to minimize the total operating cost of all committed generating units considering all physical and operational constraints. The DED problem is very complicated nonlinear optimization problem due to transmission losses, ramp-rate limits and valve-point effects constraints. Both bacterial foraging algorithm (BFA) and particle swarm optimization (PSO) methods have poor convergence characteristics. So, weighted PSO (WPSO) is employed to avoid the drawbacks of original PSO by employing the adaptive inertia weight factor. In this paper, modified BFA (MBFA) oriented with WPSO method is proposed by integrating MBFA and WPSO. By combining these two algorithms, the advantages of both of them can be extracted. This leads to get better solution of DED problem. Also, the diversity strategy is used in the proposed algorithm to solve the problem of early convergence. To prove the efficiency of the proposed method in solving the DED problem, different systems are employed. The obtained results prove the efficiency of the proposed method when comparing with other state of the art methods.

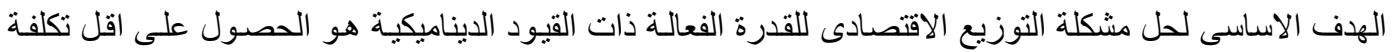

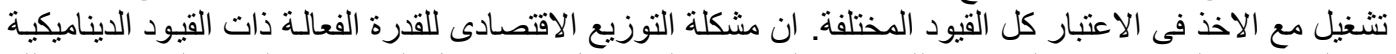

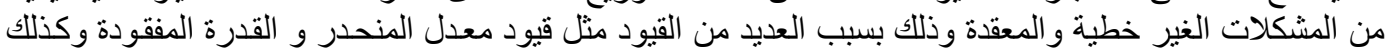

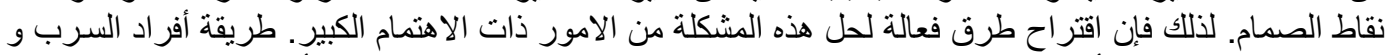

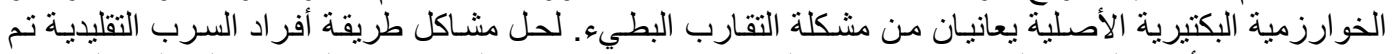

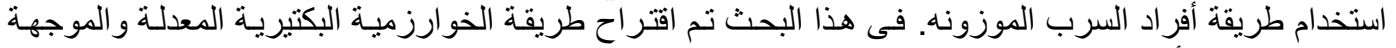

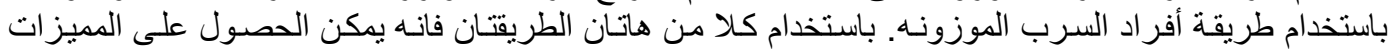

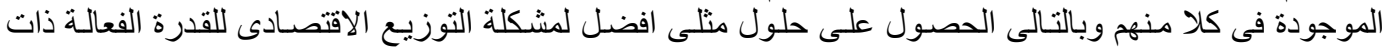

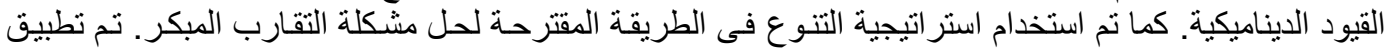

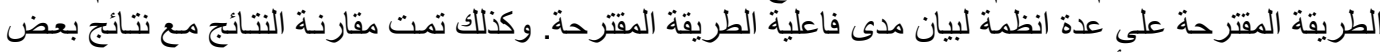

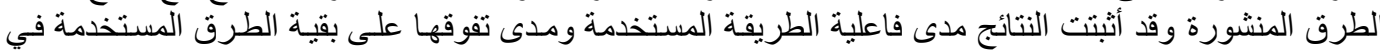

Keywords: Modified bacterial foraging algorithm, weighted particle swarm optimization, diversity strategy, dynamic economic dispatch, ramp-rate constraint.

NOMEN
$F$
$F_{i, t}$
$P_{G i, t}$
$i$
$t$
$j$
$\mathrm{n}$
$\mathrm{T}$
$a_{i}, b_{i}, c_{i}$
$\alpha_{i}, \delta_{i}$
$P_{G i, \min }$

\begin{tabular}{|c|c|}
\hline$P_{D, t}$ & The load demand at time $t$. \\
\hline$P_{\text {Loss }, t}$ & Total real power loss at time $t$. \\
\hline$B_{i j, t}$ & $\begin{array}{l}\text { Loss coefficient relating the productions of } \\
\text { units } i \text { and } j \text { at time } t\left(\mathrm{MW}^{-1}\right) \text {. }\end{array}$ \\
\hline$B_{i 0, t}$ & $\begin{array}{l}\text { Loss coefficient associated with the production } \\
\text { of unit } i \text { at time } t \text {. }\end{array}$ \\
\hline$B_{00, t}$ & Loss coefficient parameter at time $t$ (MW). \\
\hline$U R_{i}$ & Limit of ramp-up of generator $i(\mathrm{MW} / \mathrm{h})$. \\
\hline$D R_{i}$ & Limit of ramp-down of generator $i(\mathrm{MW} / \mathrm{h})$. \\
\hline$P_{G i, \max }$ & Maximum power output of generator $i(\mathrm{MW})$. \\
\hline$p_{i}^{k}$ & Position of particle $i$ at iteration $k$. \\
\hline$u_{i}^{k}$ & Velocity of particle $i$ at iteration $k$. \\
\hline
\end{tabular}




\section{E. Elattar, "Dynamic Economic Dispatch Based on MBFA oriented with WPSO"}

\begin{tabular}{|c|c|}
\hline$p_{b e s t, i}^{k}$ & $\begin{array}{l}\text { The best previous position of particle } i \text { at } \\
\text { iteration } k \text {. }\end{array}$ \\
\hline$g_{\text {best }, i}^{k}$ & $\begin{array}{l}\text { The best position among all particles in the } \\
\text { population. }\end{array}$ \\
\hline$w$ & Inertia weight parameter. \\
\hline$r_{1}, r_{2}$ & Random numbers $\epsilon[0,1]$. \\
\hline$c_{1}, c_{2}$ & PSO's acceleration coefficients. \\
\hline $\bar{w}$ & Adaptive inertia weight factor. \\
\hline$w_{\min }, w_{\max }$ & Minimum and maximum value of $w$. \\
\hline$O$ & Particle's current objective value. \\
\hline$O_{\text {avg }}$ & $\begin{array}{l}\text { Average objective value of all particles in the } \\
\text { population. }\end{array}$ \\
\hline$O_{\min }$ & $\begin{array}{l}\text { Minimum objective value of all particles in the } \\
\text { population. }\end{array}$ \\
\hline$d$ & Search space's dimension. \\
\hline$N b$ & Number of bacteria. \\
\hline$N_{l}$ & Length of a swim. \\
\hline$N_{c s}$ & Number of chemotactic steps. \\
\hline$N_{r s}$ & Number of reproduction steps. \\
\hline$N_{e s}$ & Number of elimination dispersal events. \\
\hline$P_{e s}$ & Elimination dispersal's probability. \\
\hline$C(i)$ & $\begin{array}{l}\text { Size of the step taken by each bacterium } i \text { in the } \\
\text { random direction. }\end{array}$ \\
\hline$\varphi(j)$ & Random direction of movement after a tumble \\
\hline$\theta^{i}(j, k, l)$ & $\begin{array}{l}\text { Position vector of the bacterium } i \text { in } j^{\text {th }} \\
\text { chemotactic, } k^{\text {th }} \text { reproduction and } l^{\text {th }} \text { elimination } \\
\text { dispersal step. }\end{array}$ \\
\hline$F(i, j, k, l)$ & $\begin{array}{l}\text { Objective value of bacterium } i \text { in } j^{\text {th }} \\
\text { chemotactic, } k^{\text {th }} \text { reproduction and } l^{\text {th }} \text { elimination } \\
\text { dispersal step. }\end{array}$ \\
\hline$\xi$ & Constriction coefficient. \\
\hline$b_{\text {Lbest }}$ & Best position of each bacterium. \\
\hline$b_{\text {Gbest }}$ & Global best bacteria. \\
\hline $\begin{array}{l}I(j, k, l) \\
d_{\text {attract }}\end{array}$ & Position of each bacterium of the population. \\
\hline $\begin{array}{l}w_{\text {attract }} \\
h_{\text {repellant }} \\
w_{\text {repellant }}\end{array}$ & $\begin{array}{l}\text { Coefficients which characterize the features of } \\
\text { the attractant and repellant signals. }\end{array}$ \\
\hline$\theta_{m}^{i}$ & The $m^{\text {th }}$ element of $i^{\text {th }}$ bact \\
\hline
\end{tabular}

\section{INTRODUCTION}

The economic dispatch (ED) represents one of the fundamental operation functions of the electrical power systems, particularly with the growth in cost of available fuel types [1]. The main goal of ED is to schedule the output power of all generation units. In addition, it aims to meet the load demand at a specific time with least operating cost taking into account all constraints. This makes the ED problem as a significant non-linear optimization problem [1].

There are two different types of ED. The first one called static economic dispatch (SED), while the second one is called dynamic economic dispatch (DED). In general, SED optimizes the total generation cost in a definite time without considering the connection of different operating times. On the other hand, the DED considers these connections by taking into consideration the ramp-rate limits [2].

In DED, all physical and operational constraints such as ramp-rate limits are taken into account which makes it a very complicated optimization problem [3]. Therefore getting the global optimum for this non-convex problem is a great challenge.

In literature, different algorithms are employed to get the accurate solution of the DED problem. The conventional optimization approaches such as Lagrangian relaxation (LR) [4] and dynamic programming (DP) [5] have some drawbacks. They suffer from curse of the dimensionality problem particularly in solving the problems with large scale systems. In addition, they fail to achieve the accurate solutions due to the nonlinear characteristics of the DED problem.

On the other hand and however, the artificial intelligence (AI) based methods such as differential evolution (DE) [6] and particle swarm optimization (PSO) [7] give better performance than conventional methods, they may be trapped in local optima. For this reason, researchers used hybrid methods to solve the DED problem [2, 8]. The hybrid methods have many merits. They decrease the search space. Moreover, they give an acceptable computation time in solving the DED problem. Lastly, they can deal with more constraints [2].

In recent years, swarming methods such as bacterial foraging algorithm (BFA) and PSO have been employed to get an accurate solution of the economic dispatch problem [7, 9]. To overcome the disadvantages of the conventional PSO method, weighted PSO (WPSO) is proposed and employed by merging the PSO and adaptive inertia weight factor which described in [10-12]. In the BFA, there are three essential steps. They are chemotaxis, reproduction and elimination-dispersal steps [13]. Like other AI methods, the conventional BFA may be trapped in local optima and gives poor convergence characteristics especially for nonconvex DED problem. So, these disadvantages should be overcome before using the original BFA to solve the DED problem [9].

In this paper, a hybrid WPSO and modified BFA (MBFA) is proposed to solve the problem of DED by merging the WPSO and MBFA. The WPSO method is resulting by using adaptive inertia weight factor with conventional PSO to regulate the global search, whereas, the MBFA can be derived by merging adaptive stopping criterion with original BFA. So, the proposed method has the merits of both WPSO and MBFA. In the same time it excludes the 
drawbacks of both methods. In addition, diversity strategy is employed in this paper to avoid the early convergence problem.

To prove the efficiency of the proposed method over other state of the art methods in solving the DED problem, different standard systems are serving as test systems. The contributions of this research work are: to propose a hybrid optimization method by merging MBFA with WPSO and using diversity strategy, to employ the proposed method to get the optimal solution of the DED problem and to achieve lower operating cost in comparison with other methods.

The paper is organized as follows: Section 2 shows the problem formulation of the DED problem. Section 3 describes the implementation of the proposed method. The simulation results and comparisons are shown in Section 4. Lastly, Section 5 concludes the work.

\section{MATHEMATICAL DESCRIBTION OF DYNAMIC ECONOMIC DISPATCH}

\subsection{DED Problem's Objective Function}

To achieve the objective of DED, the following quadratic objective function can be used [2]:

\section{minimize}

$F=\sum_{t=1}^{T} \sum_{i=1}^{n} F_{i, t}\left(P_{G i, t}\right)=\sum_{t=1}^{T} \sum_{i=1}^{n}\left(a_{i}+b_{i} P_{G i, t}+c_{i} P_{G i, t}^{2}\right)$

When the valve-point effect is taken into account, the total fuel cost of each generation unit can be rewritten by adding the sinusoidal function as follows [3]:

$F_{i, t}\left(P_{G i, t}\right)=a_{i}+b_{i} P_{G i, t}+c_{i} P_{G i, t}^{2}+\left|\alpha_{i} \times \sin \left(\delta_{i}\left(P_{G i, \min }-P_{G i, t}\right)\right)\right|$

\subsection{Constraints}

To solve the above objective function of DED problem, the following equal and unequal constraints are considered [2, 3].

1) System power balance:

$$
\sum_{i=1}^{n} P_{G i, t}=P_{D, t}+P_{L o s s, t}
$$

The B-coefficients formula is employed to get the transmission network losses as follows:

$P_{\text {Loss }, t}=\sum_{i=1}^{n} \sum_{i=1}^{n} P_{G i, t} B_{i j, t} P_{G j, t}+\sum_{i=1}^{n} B_{i 0, t} P_{G i, t}+B_{00, t}$ (4)
2) Generation limits:

$$
P_{G i, \min } \leq P_{G i, t} \leq P_{G i, \max }
$$

3) Up/down ramp rate limits:

$$
\begin{gathered}
U R_{i} \geq P_{G i, t}-P_{G i, t-1} \\
D R_{i} \geq P_{G i, t-1}-P_{G i, t}
\end{gathered}
$$

Taking into account the ramp rat limits, the generation limits can be rewritten as follows:

$$
\begin{aligned}
& P_{G i, t} \geq \max \left(P_{G i, \min }, P_{G i, t-1}-D R_{i}\right) \\
& \min \left(P_{G i, \max }, P_{G i, t-1}+U R_{i}\right) \geq P_{G i, t}
\end{aligned}
$$

\section{PROPOSED METHOD}

\subsection{Particle Swarm Optimization}

The original PSO begins with initial population of random solution. Each of them called particle which moves around in the search space to find the best solution. In PSO, the swarm direction of each particle depends on its own experience and the experience of closest particles [10].

In $d$-dimensional search space, the updated velocity and position of each particle of PSO can be determined as follows [14]:

$$
\begin{aligned}
u_{i}^{k+1}=w \cdot u_{i}^{k}+ & c_{1} \cdot r_{1} \cdot\left(p_{\text {best }, i}^{k}-p_{i}^{k}\right) \\
& +c_{2} \cdot r_{2} \cdot\left(g_{\text {best }, i}^{k}-p_{i}^{k}\right) \\
p_{i}^{k+1} & =p_{i}^{k}+u_{i}^{k+1}
\end{aligned}
$$

More details about conventional PSO can be found in $[10,14]$.

\subsection{Bacterial Foraging Algorithm}

The original BF algorithm has been extensively used as a global optimization method to solve many optimization problems. The foraging performance of the bacterium can be demonstrated as an optimization process where each bacterium can search for its food and avoiding noxious substances. In addition, there is a communication among bacteria [13].

As other optimization methods, the BF algorithm begins with randomly generated initial population where the number of bacteria is equal to the number of individuals in the initial population. These bacteria attempt iteratively to achieve a global optimum via four stages. They are chemotaxis, swarming, reproduction and elimination dispersal stages [9]. 


\section{E. Elattar, "Dynamic Economic Dispatch Based on MBFA oriented with WPSO"}

Firstly, each bacterium is swimming and tumbling via flagella. By altering between these two kinds of motion, the bacterium spends its lifetime. Then, the bacteria assemble into sets. They move as concentric forms with high bacterial density. After that, only fitter bacteria remain and split into two bacteria. Finally, some bacteria may be disappeared with a very small probability. More details about conventional BFA can be found in $[9,13,15]$.

\subsection{Modified BFA Oriented with Weighted PSO}

In the original PSO, choosing the parameters affects its performance. One of these parameters is the inertia weight $(w)$. This parameter controls the effect of the preceding velocity of the particle on its present one. The incorrect choice of the value of this parameter will affect the convergence speed of the algorithm. So, Liu, et al [11] combined the adaptive inertia weight factor with the PSO to regulate the global search.

By using the adaptive inertia weight factor, the parameter $w$ of the PSO will vary adaptively according to the particles' objective values as follows [11]:

$\bar{w}=\left\{\begin{array}{l}w_{\min }-\frac{\left(w_{\max }-w_{\min }\right)\left(O-O_{\min }\right)}{O_{\text {avg }}-O_{\min }}, \quad O \leq O_{\text {avg }} \\ w_{\max }, \quad O>O_{\text {avg }}\end{array}\right.$

In original BFA, the multiplication of the number of chemotactic, reproduction and elimination/dispersal steps determines the stopping criterion. This increases the execution time of the BFA.

Consequently, Farahat, et al. [16] proposed an adaptive stopping criterion to treat this problem. By employing this criterion, the number of iterations is determined according to the improvement of the objective function where the chemotaxis process stops either when the solution achieves a specific condition or when chemotactic steps equal to its maximum value [16].

In adaptive BFA (ABFA) proposed in [16], the bacteria still move randomly. This may cause delay in achieving the best solution of the optimization problem. So, random movement of bacteria can be improved in the proposed method by combining the MBFA and WPSO where the suitable direction of each bacterium will determined by its best position.

In addition, a diversity strategy based on constriction coefficient is used in this paper to avoid the premature convergence [17].

The steps of the proposed method can be summarized as follows:
Step 1: Initialize all parameters $\left(N b, N_{l}, N_{c s}, N_{r s}, N_{e s}\right.$, $\left.P_{e s}, C(i), \varphi(j), \theta(j, k, l), c_{1}, c_{2}, r_{1}, r_{2}, w_{\min }, w_{\max }\right)$ and create a random vector $\varphi(j)$ from $[-1,1]$.

Step 2: Begin the elimination dispersal loop: $l=l+l$.

Step 3: Begin the reproduction loop: $k=k+1$.

Step 4: Begin the chemotaxis loop: $j=j+1$.

- Take a chemotactic step for each bacterium (i).

- Calculate the objective function $F(i, j, k, l)$. Let $F_{\text {last }}=F(i, j, k, l)$ therefore, the best value can be found.

- For $i=1, \ldots, N b$, both tumbling and swimming' decision can be taken as follows:

Tumble: the best position of each bacterium and the global best position will decide the direction as follows:

$$
\begin{gathered}
\phi(j+1)=\xi\left(\bar{w} \cdot \phi(j)+c_{1} \cdot r_{1} \cdot\left(b_{\text {Lbest }}-b_{c}\right)\right. \\
\left.\left.+c_{2} \cdot r_{2} \cdot\left(b_{\text {Gbest }}-b_{c}\right)\right)\right)
\end{gathered}
$$$$
\text { where }
$$

$$
\xi=\frac{2}{\left|2-c_{1}-c_{2}-\sqrt{\left(c_{1}+c_{2}\right)^{2}-4\left(c_{1}+c_{2}\right)}\right|} .
$$

and $\mathrm{c}_{1}+\mathrm{c}_{2}>4$.

Move: Let:

$$
\theta^{i}(j+1, k, l)=\theta^{i}(j, k, l)+C(i) \phi(j)^{\prime}
$$

- Compute: $F(i, j+1, k, l)$, then let:

$$
\begin{aligned}
F(i, j+1, k, l) & =F(i, j+1, k, l)+ \\
+ & F_{c c}\left(\theta^{i}(j+1, k, l), I(j+1, k, l)\right) \\
= & F(i, j+1, k, l)+ \\
+ & \sum_{i=1}^{S}\left[-d_{\text {attract }} \exp \left(-w_{\text {atract }} \sum_{m=1}^{d}\left(\theta_{m}-\theta_{m}^{i}\right)^{2}\right)\right] \\
+ & \sum_{i=1}^{S}\left[-h_{\text {repellant }} \exp \left(-w_{\text {repellant }} \sum_{m=1}^{d}\left(\theta_{m}-\theta_{m}^{i}\right)^{2}\right)\right]^{\prime}
\end{aligned}
$$

- Loop of swimming: Let $q=O$ ( $q$ is swim length's counter).

While q $<N_{l}$

Let $\mathrm{q}=q+1$

- If $F(i, j+1, k, l)<F_{\text {last }}$, then let:

$$
F_{\text {last }}=F(i, j+1, k, l) \text {. }
$$

- Let

$$
\theta^{i}(j+1, k, l)=\theta^{i}(j, k, l)+C(i) \phi(j)^{\prime}
$$

- At that moment, compute the new objective function $F(i, j+1, k, l)$ using (16).

- Else, let $q=N_{l}$ (finishing the while statement). 


\section{E. Elattar, "Dynamic Economic Dispatch Based on MBFA oriented with WPSO"}

- If $i \neq N b$, proceed to the following bacterium.

- After that the best objective value gotten $\left(F_{\text {best }}(j)\right)$ can be calculated.

- The difference $(f)$ in the objective value obtained in the current chemotactic step can be computed as follows [16]:

- $f(j)=F_{\text {best }}(j)-F_{\text {best }}(j-1)$.

- If $j>N_{c s} / 2$.

- If $|f(j)-f(j-b)|<\varepsilon ; \quad b=1,2, \ldots, b_{m}$ and $b_{m}<N_{c s} / 2$.

- $j=N c$ (stop chemotactic steps).

Step 5: If $j<N_{c s}$, return to step 4.

Step 6: Reproduction: each bacterium's health can be computed as:

$$
F_{\text {health }}^{i}=\sum_{j=1}^{N_{c}+1} F(i, j, k, l)
$$

Arrange the bacteria in an ascending order where bacteria with highest health will die while the residual bacteria reproduce.

Step 7: If $k<N_{r s}$, return to step 3, otherwise, go to step 8 .

Step 8: Elimination-Dispersal: a few bacteria are removed with small probability $\left(P_{e s}\right)$. So, the number of bacteria in the population is kept constant.

Step 9: If $l<N e s$, then return to step 2; otherwise end.

\section{SIMULATION RESULTS}

The proposed method is evaluated by applying it on three commonly used test systems. These systems are 5 thermal generation units system, 10 thermal generation units system and 30 thermal generation units system. The required data of each system (Fuel cost's coefficients for each thermal generator unit, valve-point's coefficients for each thermal generator unit, minimum and maximum output power of each thermal generator unit, limit of ramp-up and rampdown of each thermal generator unit, B - matrix coefficients and load profile) is collected from Ref. [18]. To sake the fair comparison with other methods, valve-point effect is considered for the three test systems, while the transmission losses are considered only for the first two systems. Table 1 shows the details of these three commonly used test systems.

The proposed method is executed in personal computer with Pentium 4 processor, $2.8 \mathrm{GHz}$ clock frequency and $4 \mathrm{~GB}$ of RAM using MATLAB R2012a. In each system, 100 independent runs were carried out with random initial values for each run and results (minimum, average, maximum) were obtained.

In order to execute the proposed method, some parameters should be adjusted first. These parameters affect not only the speed of convergence but also solution's quality. In this work, these parameters are chosen using empirical tests by solving the DED problem with different values of the parameters. Table 2 shows the best values of the parameters used in each system.

\subsection{Test System 1: 5-Unit system}

The best solution obtained for this system is shown in Table 3. The performance of the proposed method is compared with adaptive particle swarm optimization (APSO) algorithm [20], simulated annealing (SA) algorithm [19], artificial immune system (AIS) [21], Maclaurin series-based Lagrangian (MSL) method [22], GA [23], PSO [23], artificial bee colony (ABC) algorithm [23], time varying acceleration coefficients improved particle swarm optimization (TVAC-IPSO) [24], hybrid immune-genetic algorithm (HIGA) [2] and hybrid genetic algorithm and bacterial foraging (HGABF) [25]. This comparison is shown in Table 4. The results of Table 4 prove that the proposed method yields better results than other methods.

To test the superiority of the solution, the standard deviation (SD) from 100 independent runs by the proposed method is obtained. The value of this SD is equal to $\$ 13.63$. This shows a small range of variation the total cost achieved by the proposed method. This is evidence of the robustness of the proposed method.

\subsection{Test System 2: 10-Unit system}

The performance of the proposed method is compared with evolutionary programming (EP) [26], hybrid evolutionary programming and sequential quadratic programming (EP-SQP) [26], modified EPSQP (MHEP-SQP) [26], GA [23], PSO [23], ABC [23], improved PSO (IPSO) [27], Enhanced crossentropy (ECE) [28], AIS [21], enhanced bee swarm optimization (EBSO) [8], HIGA [2], enhanced adaptive particle swarm optimization (EAPSO) [29] and HGABF [25]. This comparison is shown in Table 5. These results demonstrate that the proposed method is more efficient than other methods.

The obtained SD from among 100 independent runs in this system is $\$ 199.87$ which emphasizes again the robustness of the proposed method.

\subsection{Test System 3: 30-Unit system}

Many state of the art methods are used in comparison in this test system. These methods are EP [30], EPSQP [30], MHEP-SQP [27], IPSO [28], improved chaotic (ICPSO) [31], harmony search algorithm 
E. Elattar, "Dynamic Economic Dispatch Based on MBFA oriented with WPSO"

Table (1), The Details of the Three Test Systems.

\begin{tabular}{|c|c|c|c|}
\hline & System 1 & System 2 & System 3 \\
\hline $\begin{array}{c}\text { Number of generation } \\
\text { units }\end{array}$ & 5 & 10 & 30 \\
\hline valve-point effects & Yes & Yes & Yes \\
\hline $\begin{array}{l}\text { Constraints used to sake } \\
\text { the fair comparison with } \\
\text { other methods }\end{array}$ & $\begin{array}{l}\text { - Transmission losses. } \\
\text { - Generation limits. } \\
\text { - Ramp-rate limits }\end{array}$ & $\begin{array}{l}\text { - Transmission losses. } \\
\text { - Generation limits. } \\
\text { - Ramp-rate limits }\end{array}$ & $\begin{array}{l}\text { - Generation limits. } \\
\text { - Ramp-rate limits }\end{array}$ \\
\hline Dispatch horizon & $\begin{array}{l}\text { One day with periods of } \\
1 \mathrm{~h} .\end{array}$ & $\begin{array}{l}\text { One day with periods of } \\
\qquad 1 \mathrm{~h} .\end{array}$ & $\begin{array}{l}\text { One day with periods } \\
\text { of } 1 \mathrm{~h} .\end{array}$ \\
\hline $\begin{array}{l}\text { Data of the system } \\
\text { (coefficients of each } \\
\text { generator, minimum and } \\
\text { maximum output power of } \\
\text { each generator and limit of } \\
\text { ramp-up and down of each } \\
\text { generator) }\end{array}$ & $\begin{array}{l}\text { Data of this system is } \\
\text { collected from Ref. [18] }\end{array}$ & $\begin{array}{l}\text { Data of this system is } \\
\text { collected from Ref. [18] }\end{array}$ & $\begin{array}{c}\text { Data are gained by } \\
\text { tripling the data of } \\
\text { system } 2 .\end{array}$ \\
\hline B - matrix coefficients & $\begin{array}{l}\text { These coefficients are } \\
\text { given in Ref. [18] }\end{array}$ & $\begin{array}{l}\text { These coefficients are } \\
\text { given in Ref. [18] }\end{array}$ & $\begin{array}{l}\text { Transmission losses are } \\
\text { neglected to sake the } \\
\text { fair comparison with } \\
\text { other methods. }\end{array}$ \\
\hline Load profile & $\begin{array}{l}\text { Hourly load values are } \\
\text { extracted from Ref. [2]. }\end{array}$ & $\begin{array}{l}\text { Hourly load values are } \\
\text { extracted from Ref. [2]. }\end{array}$ & $\begin{array}{l}\text { Hourly load values are } \\
\text { extracted from Ref. [2]. }\end{array}$ \\
\hline
\end{tabular}

Table (2), Parameters of the Proposed Method for Each Test System.

\begin{tabular}{|c|c|c|c|}
\hline & System 1 & System 2 & System 3 \\
\hline Parameters of MBFA & $\begin{array}{l}N b=100, N_{c s}=25, N_{l}= \\
4, N_{r s}=4, N_{e s}=2, P_{e s}= \\
0.25 \text { and } C(i)=0.1\end{array}$ & $\begin{array}{l}N b=120, N_{c s}=30, N_{l}= \\
4, N_{r s}=4, N_{e s}=2, P_{e s}= \\
0.25 \text { and } C(i)=0.1\end{array}$ & $\begin{array}{l}N b=120, N_{c s}=50, N_{l}= \\
4, N_{r s}=4, N_{e s}=2, P_{e s}= \\
0.25 \text { and } C(i)=0.1\end{array}$ \\
\hline Parameters of WPSO & $\begin{array}{l}c_{1}=c_{2}=2.1, \quad w_{\min }=0.2 \text { and } \\
w_{\text {max }}=0.9\end{array}$ & $\begin{array}{l}c_{1}=c_{2}=2.1, \quad w_{\min }=0.4 \text { and } \\
w_{\max }=1.2\end{array}$ & $\begin{array}{l}c_{1}=c_{2}=2.1, \quad w_{\min }=0.4 \text { and } \\
w_{\max }=1.2\end{array}$ \\
\hline
\end{tabular}

Table (3), The Best Solution Obtained for System 1 (5 Unit System).

\begin{tabular}{|c|c|c|c|c|c|c|c|c|c|c|c|c|c|}
\hline$\stackrel{\Xi}{\Xi}$ & $\mathbf{P}_{1}$ & $\mathbf{P}_{2}$ & $\mathbf{P}_{3}$ & $\mathbf{P}_{4}$ & $\mathbf{P}_{5}$ & Loss & $\stackrel{\Xi}{\Xi}$ & $\mathbf{P}_{1}$ & $\mathbf{P}_{2}$ & $\mathbf{P}_{3}$ & $\mathbf{P}_{4}$ & $\mathbf{P}_{5}$ & Loss \\
\hline 1 & 10.00 & 20.00 & 30.03 & 123.08 & 229.80 & 2.91 & 13 & 62.60 & 99.14 & 111.30 & 211.43 & 230.08 & 10.55 \\
\hline 2 & 10.00 & 20.00 & 54.44 & 124.58 & 229.21 & 3.23 & 14 & 48.52 & 98.95 & 111.30 & 211.22 & 230.08 & 10.07 \\
\hline 3 & 10.00 & 28.55 & 87.10 & 124.23 & 230.02 & 4.90 & 15 & 36.66 & 98.12 & 111.30 & 186.97 & 230.08 & 9.13 \\
\hline 4 & 11.38 & 56.51 & 111.99 & 125.22 & 231.32 & 6.42 & 16 & 10.00 & 98.55 & 111.30 & 137.20 & 230.11 & 7.16 \\
\hline 5 & 10.12 & 86.51 & 110.32 & 126.21 & 231.30 & 6.46 & 17 & 10.00 & 86.65 & 111.41 & 126.41 & 230.15 & 6.62 \\
\hline 6 & 10.12 & 97.33 & 110.32 & 167.00 & 231.12 & 7.89 & 18 & 10.21 & 101.1 & 107.27 & 167.25 & 230.08 & 7.91 \\
\hline 7 & 10.12 & 100.15 & 83.46 & 210.09 & 231.02 & 8.84 & 19 & 11.30 & 99.56 & 110.55 & 211.44 & 230.08 & 8.93 \\
\hline 8 & 13.27 & 100.99 & 107.26 & 211.43 & 230.08 & 9.03 & 20 & 41.73 & 98.68 & 131.80 & 211.44 & 230.38 & 10.03 \\
\hline 9 & 42.26 & 104.84 & 111.54 & 211.43 & 230.08 & 10.15 & 21 & 38.06 & 100.5 & 109.92 & 211.44 & 230.02 & 9.89 \\
\hline 10 & 62.31 & 99.11 & 111.54 & 211.43 & 230.11 & 10.50 & 22 & 10.00 & 98.88 & 109.92 & 162.91 & 231.17 & 7.88 \\
\hline 11 & 75.00 & 103.14 & 111.30 & 211.43 & 230.08 & 10.95 & 23 & 10.00 & 98.30 & 109.92 & 126.00 & 188.70 & 5.92 \\
\hline 12 & 73.99 & 98.78 & 137.03 & 211.43 & 230.08 & 11.31 & 24 & 10.00 & 97.83 & 92.45 & 126.00 & 141.22 & 4.50 \\
\hline
\end{tabular}


E. Elattar, "Dynamic Economic Dispatch Based on MBFA oriented with WPSO"

Table (4), Fuel Cost Comparison for System 1 (5-unit system).

\begin{tabular}{|l|c|c|c|}
\hline \multicolumn{1}{|c|}{ Method } & Minimum Cost (\$) & Average Cost (\$) & Maximum Cost (\$) \\
\hline MSL [20] & 49216.81 & NA $^{*}$ & NA \\
\hline SA [19] & 47356.00 & NA & NA \\
\hline GA [23] & 44862.42 & 44921.76 & 45893.95 \\
\hline APSO [20] & 44678.00 & NA & NA \\
\hline AIS [21] & 44385.43 & 44758.84 & 45553.77 \\
\hline PSO [23] & 44253.24 & 45657.06 & 46402.52 \\
\hline ABC [23] & 44045.83 & 44064.73 & 44218.64 \\
\hline TVAC-IPSO [24] & 43136.56 & 43185.66 & 43302.23 \\
\hline HIGA [2] & 43125.37 & 43162.24 & 43259.35 \\
\hline HGABF [ 25] & 41574.80 & 41599.68 & 41652.56 \\
\hline Proposed method & 40160.54 & 40182.21 & 40220.30 \\
\hline
\end{tabular}

Table (5), Fuel Cost Comparison for System 2 (10-unit system).

\begin{tabular}{|l|c|c|c|}
\hline \multicolumn{1}{|c|}{ Method } & Minimum Cost (\$) & Average Cost (\$) & Maximum Cost (\$) \\
\hline EP [26] & 1054685 & 1057323 & NA \\
\hline EP-SQP [26] & 1052668 & 1053771 & NA \\
\hline GA [23] & 1052251 & 1058041 & 1062511 \\
\hline MHEP-SQP [26] & 1050054 & 1052349 & NA \\
\hline PSO [23] & 1048410 & 1052092 & 1057170 \\
\hline IPSO [27] & 1046275 & 1048145 & NA \\
\hline AIS [21] & 1045715 & 1047050 & 1048431 \\
\hline ECE [28] & 1043989.154 & 1044470.0849 & NA \\
\hline ABC [23] & 1043381 & 1044963 & 1046805 \\
\hline HIGA [2] & 1041087.802 & 1042980.147 & 1044926.653 \\
\hline EBSO [8] & 1038915 & 1039188 & 1039272 \\
\hline EAPSO [29] & 1037898 & 1038109 & 1038238 \\
\hline HGABF [25] & 1036507 & 1037068 & 1038092 \\
\hline Proposed method & 1035573 & 1036076 & 1036762 \\
\hline
\end{tabular}

Table (6), Fuel Cost Comparison for System 3 (30-unit system).

\begin{tabular}{|l|c|c|c|}
\hline \multicolumn{1}{|c|}{ Method } & Minimum Cost $(\$)$ & Average Cost (\$) & Maximum Cost (\$) \\
\hline EP [30] & 3164531 & 3200171 & NA \\
\hline EP-SQP [30] & 3159204 & 3169093 & NA \\
\hline MHEP-SQP [26] & 3151445 & 3157438 & NA \\
\hline DGPSO [33] & 3148992 & 3154438 & NA \\
\hline HS [32] & 3143253.84 & NA & NA \\
\hline IPSO [27] & 3090570 & 3090570 & NA \\
\hline CE [28] & 3086109.595 & 3088869.8572 & NA \\
\hline ECE [28] & 3084649.032 & 3087847.1893 & NA \\
\hline ICPSO [31] & 3064497 & 3071588 & NA \\
\hline HHS [32] & 3057313.39 & NA & NA \\
\hline HIGA [2] & 3055435.068 & 3058126.233 & 3066754.92 \\
\hline EAPSO [29] & 3054961 & 3055257 & 3055641 \\
\hline EBSO [8] & 3054001 & 3054697 & 3055944 \\
\hline HGABF [25] & 3050235 & 3051291 & 3053567 \\
\hline Proposed method & 3047150 & 3048277 & 3050349 \\
\hline
\end{tabular}

* NA: means that this data is not available in the reference. 
(HS) [32], hybrid swarm intelligence-based harmony search (HHS) [32], deterministically guided PSO (DGPSO) [33], cross entropy (CE) [29], enhanced cross entropy (ECE) [29], HIGA [2], EAPSO [30], EBSO [8] and HGABF) [26]. This comparison is shown in Table 6 . In this test system, the gained SD among 100 independent runs is $\$ 502.08$. This proves the robustness of the proposed method despite the number of generation units.

From the results in tables 4 - 6 which depicted in figures 1-3, one can notice that the proposed method is compared with different stat of the art methods based on minimum, average and maximum fuel costs. The stat-of-the-art methods' results are obtained from their references.

For the three test systems, the maximum fuel cost obtained using the proposed method is better than the minimum fuel cost obtained from all methods except HGABF method in system 2 and 3. This shows the superiority of the proposed method over other methods for different systems with different number of generation units. Also, the results prove the stability of the proposed method which evident from the small difference between minimum and maximum fuel cost whatever the number of generation units.
As we know, the execution time is highly affected by many factors such as, coding the algorithm and the configuration of the computer. So, in this paper, the execution time of the proposed method and HGABF method is compared using the same computer configuration. The execution times of other methods are not used in the comparison because they may have employed different computer configuration. Figure 4 shows the results of this comparison. These results demonstrate the high convergence speed for the proposed method over HGABF and original BFA methods. This is due to employing the adaptive stopping criterion in the proposed method which reduces the number of chemotaxis steps. The real life DED problem is solved offline. This makes the execution time of the proposed method (several minutes) is suitable to solve this problem.

\section{CONCLUSION}

In this work, an optimization method is proposed to solve the DED problem. The proposed method is resulting by merging BFA, adaptive stopping criterion, PSO, adaptive inertia weight factor and diversity strategy. This leads to avoid the drawbacks of these methods and gain the merits of them. The

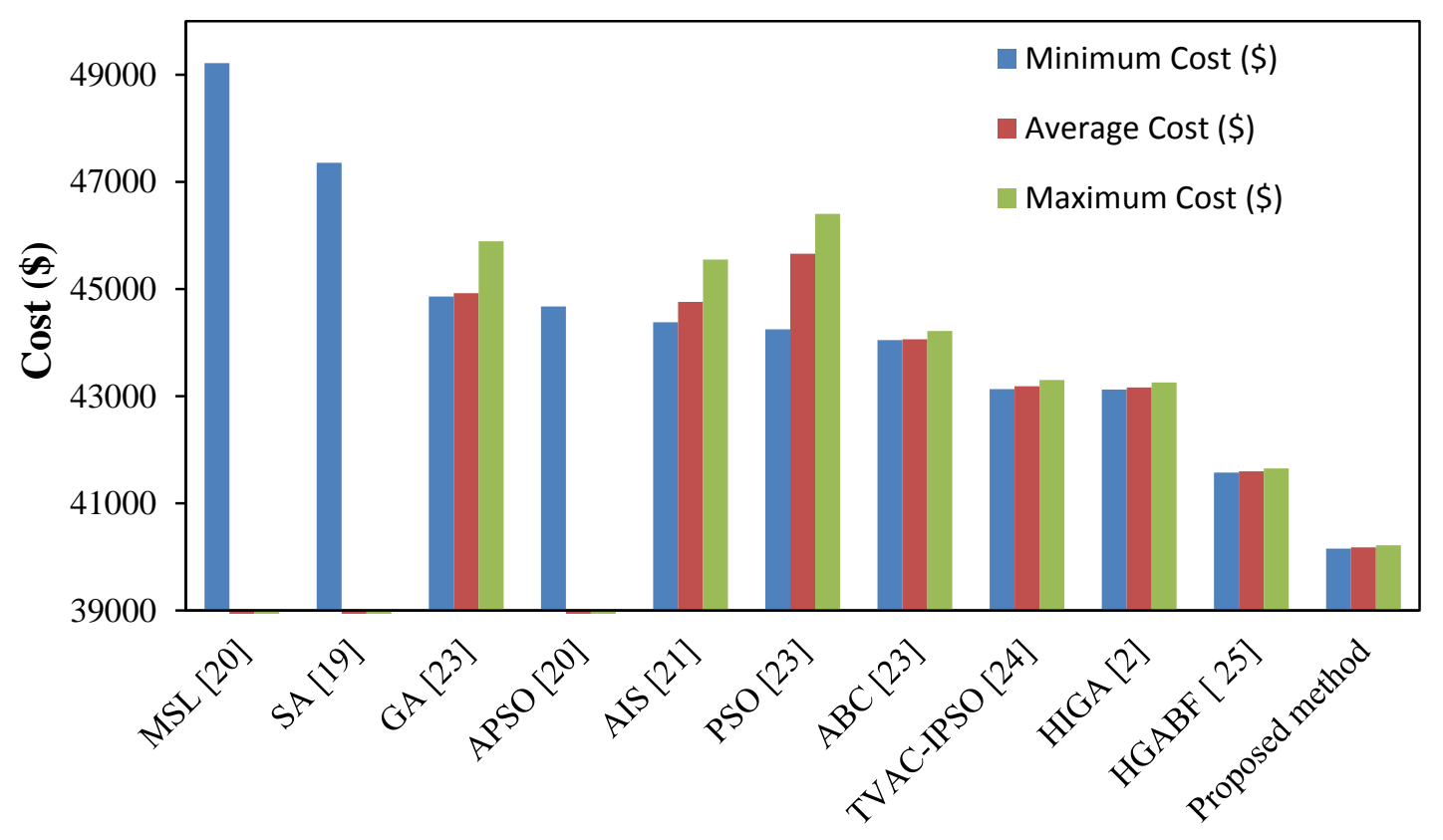

Fig. 1, Fuel Cost Comparison for System 1 (5-unit system). 


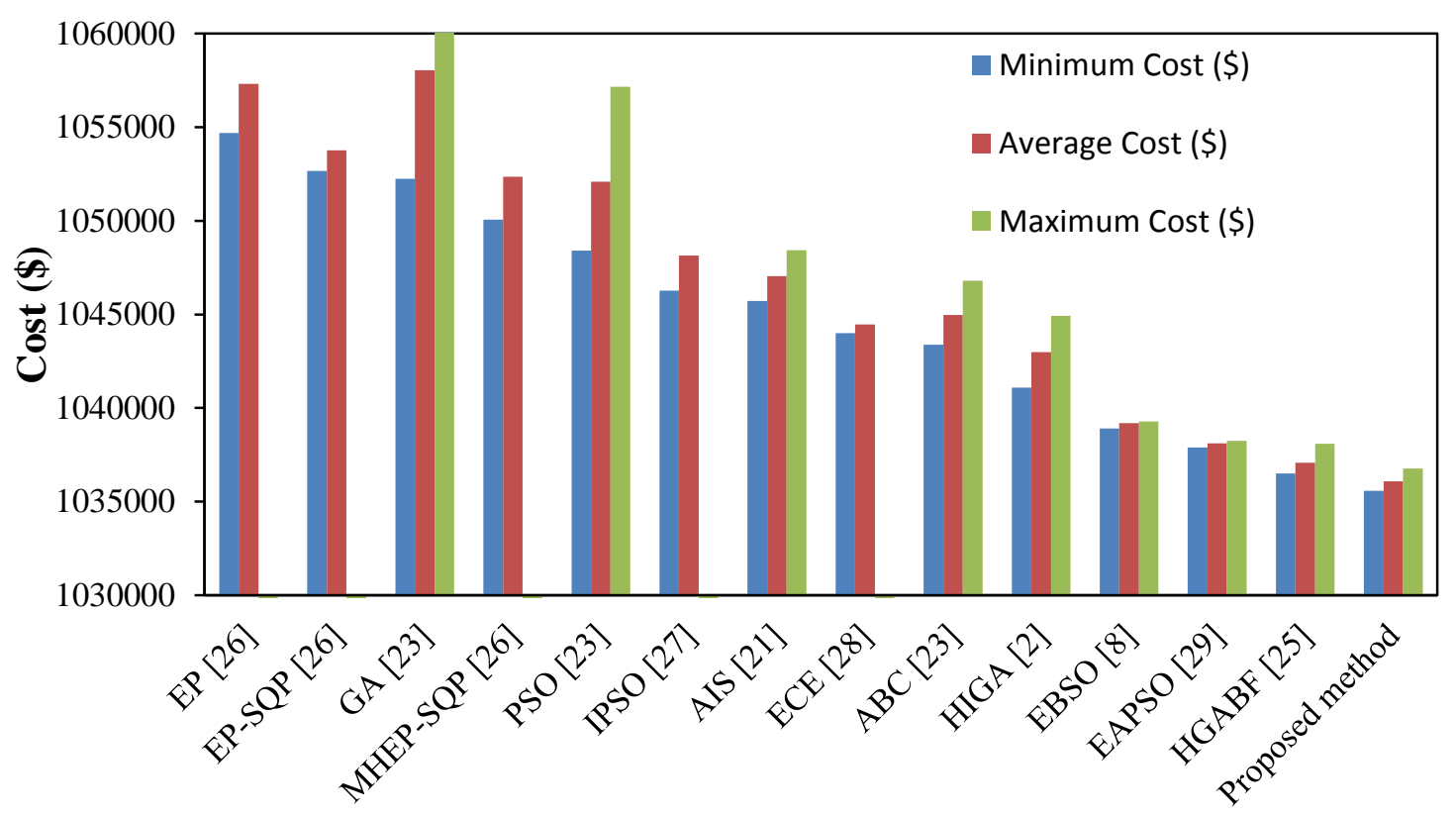

Fig. 2, Fuel Cost Comparison for System 2 (10-unit system).

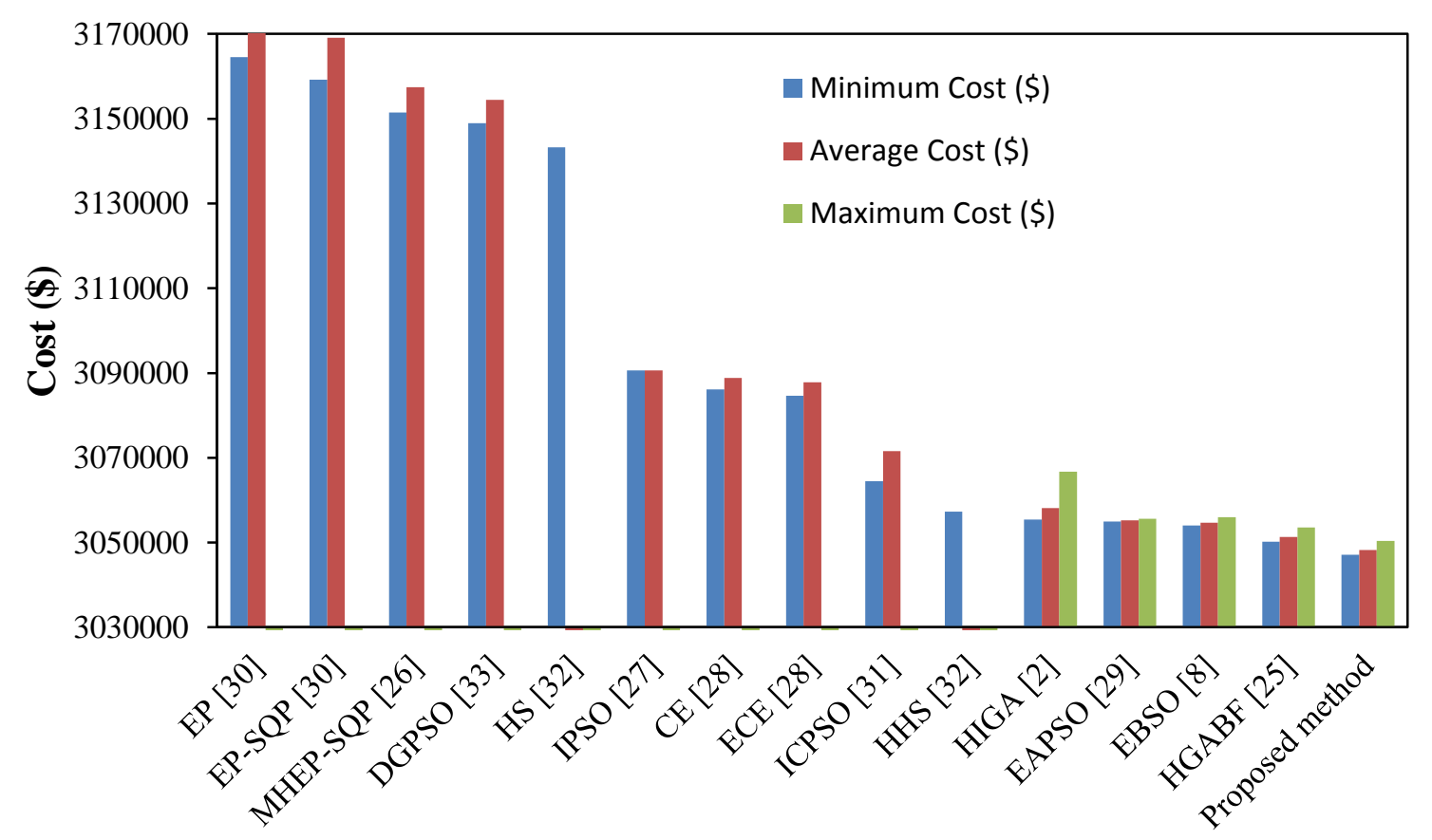

Fig. 3, Fuel Cost Comparison for System 3 (30-unit system). 


\section{E. Elattar, "Dynamic Economic Dispatch Based on MBFA oriented with WPSO"}

feasibility and effectiveness of the proposed method have been confirmed using three test systems. The simulation results were compared with some of state of the art methods. The results show the superiority of the proposed method over these state of the art methods for solving the DED problem.

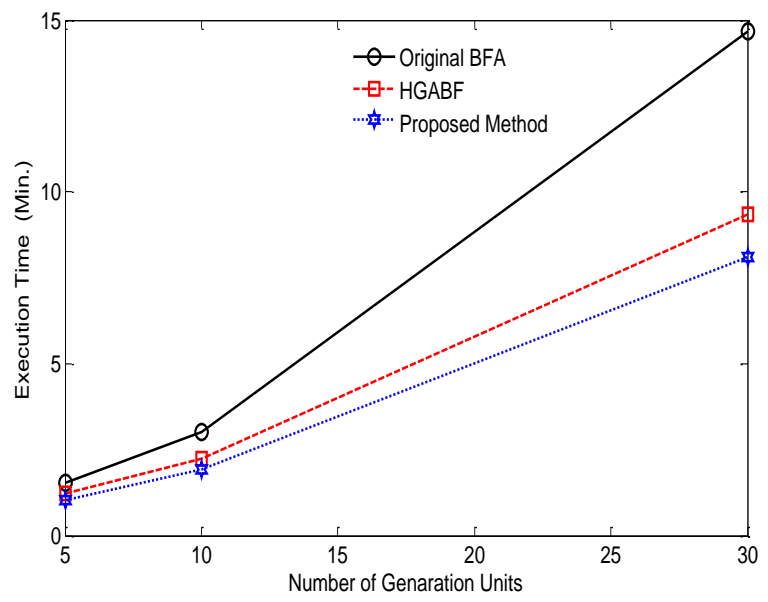

Fig. 4. Execution time for different methods

Also, they confirmed the capability of the proposed method to get the global solution of the DED problem.

\section{REFERENCES}

[1] H. Xing, Y. Mou, M. Fu and Z. Lin, "Distributed bisection method for economic power dispatch in smart grid", IEEE Transactions on Power Systems, vol. 30, no. 6, pp. 3024-3035, 2015.

[2] B. Ivatloo, A. Rabiee and A. Soroudi, "Nonconvex dynamic economic power dispatch problems solution using hybrid immune-genetic algorithm", IEEE Transactions on Power Systems, vol. 7, no. 4, pp. 777785, 2013.

[3] M. Wang, H. Gooi, S. Chen and S. Lu, "A mixed integer quadratic programming for dynamic economic dispatch with valve point effect", IEEE Transactions on Power Systems, vol. 29, no. 5, pp. 2097-2106, 2014.

[4] K. Hindi and M. Ghani, "Dynamic economic dispatch for large scale power systems: a Lagrangian relaxation approach", International Journal of Electrical Power Energy System, vol. 13, no. 1, pp. 51-56, 1991.

[5] H. Zhong, Q. Xia, Y. Wang and C. Kang, "Dynamic economic dispatch considering transmission losses using quadratically constrained quadratic program method", IEEE Transactions on Power Systems, vol. 28, no. 3, pp. 2232-2241, 2013.

[6] Y. Lu, J. Zhou, H. Qin, Y. Wang and Y. Zhang, "Chaotic differential evolution methods for dynamic economic dispatch with valve-point effects", Engineering Application of Artificial Intelligence, vol. 24, no. 12, pp. 378-387, 2011.

[7] J. Sun, V. Palade, X. Wu, W. Fang and Z. Wang, "Solving the power economic dispatch problem with generator constraints by random drift particle swarm optimization", IEEE Transactions on Industrial Informatics, vol. 10, no. 1, pp. 222-232, 2014.
[8] T. Niknam and F. Golestaneh, "Enhanced bee swarm optimization algorithm for dynamic economic dispatch", IEEE Systems Journal, vol. 7, no. 4, pp. 754$762,2013$.

[9] Z. Zakaria, T. Rahman and E. Hassan, "Economic load dispatch via an improved Bacterial Foraging Optimization", $8^{\text {th }}$ IEEE International Power Engineering and Optimization Conference (PEOCO), 2014, pp. 380-385.

[10] C. Jiejin, M. Xiaoqian, L. Lixiang and P. Haipeng, "Chaotic particle swarm optimization for economic dispatch considering the generator constraints", Energy Conversion and Management, vol. 48, pp. 645-653, 2007.

[11] B. Liu, L. Wang, Y. Jin, F. Tang and D. Huang, "Improved particle swarm optimization combined with chaos", Solitons Fractals, vol. 25, no. 5, pp.1261-1271, 2005.

[12] Y. Zhu and et. al., "Hierarchical economic load dispatch based on chaotic-particle swarm optimization", Ninth International Conference on Natural Computation (ICNC), Jul. 23-25, 2013, pp. 517-521.

[13] K. Passino, "Biomimicry of bacterial foraging for distributed optimization and control", IEEE Control Systems Magazine, vol. 22, no. 3, pp. 52-67, 2002.

[14] J. Kennedy and R. Eberhart, "Particle swarm optimization", In Proc. IEEE Int. Conf. Neural Networks (ICNN'95), Vol. IV, Perth, Australia,1995, pp. 1942-1948.

[15] A. Boughelala, L Xiaoming and S. Leghmizi, "An adaptive channel equalizer using bacterial foraging oriented by particle swarm optimization strategy", 3rd International Conference on Computer Research and Development (ICCRD), March 2011, China, pp. 24-29.

[16] I. Farhat, M. El-Hawary, "Dynamic adaptive bacterial foraging algorithm for optimum economic dispatch with valve-point effects and wind power", IET Generation, Transmission and Distribution, vol. 4, no. 9, pp. 989-999, 2010.

[17] M. Clerc and J. Kennedy, "The particle swarm explosion, stability, and convergence in a multidimensional complex space," IEEE Transactions on Evolutionary Computation, vol. 6, pp. 58-73, 2002.

[18] B. Ivatloo, A. Rabiee, A. Soroudi and M. Ehsan, "Imperialist competitive algorithm for solving nonconvex dynamic economic power dispatch", Energy (Elsevier), vol. 44, pp. 228-240, 2012.

[19] C. Panigrahi, P. Chattopadhyay, R. Chakrabarti and M. Basu, "Simulated annealing technique for dynamic economic dispatch", Electric Power Component and Systems, vol. 34, no. 5, pp. 577-586, 2006.

[20] B. Panigrahi, V. Pandi and S. Das, "Adaptive particle swarm optimization approach for static and dynamic economic load dispatch", Energy Conversion and Management, vol. 49, no. 6, pp. 1407-1415, 2008.

[21] S. Hemamalini and S. Simon, "Dynamic economic dispatch using artificial immune system for units with valve-point effect", Electrical Power Energy Systems, vol, 33, no. 4, pp. 868-874, 2011.

[22] S. Hemamalini and S. Simon, "Dynamic economic dispatch using Maclaurin series based Lagrangian method", Energy Conversion and Management, vol. 51, no. 11, pp. 2212-2219, 2010. 
[23] S. Hemamalini and S. Simon, "Dynamic economic dispatch using artificial bee colony algorithm for units with valve-point effect", European Transaction of Electrical Power, vol. 21, no. 1, pp. 70-81, 2011.

[24] B. Ivatloo, A. Rabiee and M. Ehsan, "Time-varying acceleration coefficients IPSO for solving dynamic economic dispatch with non-smooth cost function", Energy Conversion and Management, vol. 56, pp. 175183, 2012.

[25] E. E. Elattar, "A hybrid genetic algorithm and bacterial foraging approach for dynamic economic dispatch problem," International Journal of Electrical Power and Energy Systems (Elsevier), vol. 69, pp. 1826, 2015.

[26] T. Victoire and V. Jeyakumar, "A modified hybrid EP-SQP approach for dynamic dispatch with valvepoint effect", Electrical Power and Energy Systems, vol. 27, no. 8, pp. 594-601, 2005.

[27] X. Yuan, A. Su, Y. Yuan, H. Nie and L. Wang, "An improved PSO for dynamic load dispatch of generators with valve-point effects", Energy, vol. 34, no.1, pp. 6774, 2009.

[28] A. Selvakumar, "Enhanced cross-entropy method for dynamic economic dispatch with valve-point effects", International Journal of Electric Power and Energy Systems, vol. 33, no. 3, pp. 783-790, 2011.

[29] T. Niknam and F. Golestaneh, "Enhanced adaptive particle swarm optimisation algorithm for dynamic economic dispatch of units considering valve-point effects and ramp rates", IET Gen. Transm. And Distrib. vol. 6, no. 5, pp. 424-435, 2012.

[30] P. Attaviriyanupap, H. Kita, E. Tanaka and J. Hasegawa, "A hybrid EP and SQP for dynamic economic dispatch with non-smooth fuel cost function", IEEE Transaction on Power Systems, vol. 17, no. 2, pp. 411-416, 2002.

[31] Y. Wang, J. Zhou, H. Qin and Y. Lu, "Improved chaotic particle swarm optimization algorithm for dynamic economic dispatch problem with valve-point effects", Energy Conversion and Management, vol. 51, no. 12, pp. 2893-28900, 2010.

[32] V. Pandi and B. Panigrahi, "Dynamic economic load dispatch using hybrid swarm intelligence based harmony search algorithm", Expert Syst. Appl., vol. 38, no. 7, pp. 8509-8514, 2011.

[33] T. Victoire and A. Jeyakumar, "Deterministically guided PSO for dynamic dispatch considering valvepoint effect", Electric Power System Research, vol. 37, no. 3, pp. 313-322, 2005. 